\title{
Analisi delle antibiotico-resistenze di Gram-negativi isolati da pazienti con infezioni del tratto urinario afferenti al Polo Oncologico e Dermatologico I.F.O.
}

\author{
Maria Teresa Gallo', Grazia Prignano', Mauro Belardi', Karim Donato', \\ Gian Piero Testore ${ }^{2}$, Fabrizio Ensoli'
}

'Laboratorio di Patologia Clinica e Microbiologia, Istituto San Gallicano IRCCS, Roma; ${ }^{2}$ Dipartimento di Sanità Pubblica e Biologia Cellulare, Università di Tor Vergata, Roma

Key words: Urinary tract infections, Gram-Negative Bacteria, Bacterial drug resistence

Analysis of antibiotic-resistance among Gram-negative pathogens isolated from the urinary tract of patients at the Oncology/Dermatology Centre in Rome

\section{SUMMARY}

Introduction. Urinary tract infections represent a worlwide problem due to their prevalence among nosocomial infections and to the increasing frequency of antibiotic resistance among the Gram-negative pathogens. Knowledge of the antimicrobial resistance patterns according to local epidemiology is essential for providing clinically appropriate therapy for urinary tract infection. In the present study we analysed the drug resistence pattern of Gram negative bacteria isolated from urine samples of patients with urinary tract infections at the Microbiological Laboratory of I.F.O., Rome.

Materials and Methods. A total 3650 isolates have been collected during three years (2000-2003). Isolates were subcultured to Agar blood-Mac-Conckey and analysed in the Vitek I for identification and drug susceptibility.

Results. As expected, Escherichia coli was found to be the main cause of urinary tract infection (73\%). Proteus mirabilis, Klebsiella pneumoniae, and Pseudomonas aeruginosa, were less frequently isolated $(9 ., 6 \%, 8.9 \%$ and $4.5 \%$, respectively).

The E. coli had the highest resistance rate to ampicillin (38\%), followed by trimethoprim-sulphamethoxazole (22\%) and ciprofloxacine (16\%), while nitrofurantoin susceptibility of the $E$. coli isolates was still at acceptable levels $(98 \%)$. The P. mirabilis isolates were found to be resistant to amoxycillin (35\%), to trimethoprimsulphamethoxazole (34\%). The P. aeruginosa isolates were found to be resistant to ciprofloxacina and imipenem ( $48 \%$ and $28 \%$ respectively).

Conclusions. The increased drug resistance developed by bacteria consistently due to previous treatments with several antimicrobial drugs makes it essential the culture and susceptibility testing in genitourinary tract infection before instituting antimicrobial therapy. Empirical antimicrobial therapy should be avoided or reassessed by 48 to 72 hours after initation based on preterapy culture results.

\section{INTRODUZIONE}

Le infezioni del tratto urinario, rappresentano un problema terapeutico soprattutto in relazione alla loro prevalenza nell'ambito delle infezioni nosocomiali o acquisite in comunità (14). Sono causate prevalentemente da E. coli e altri Gram-negativi, i quali rappresentano 1'80\% degli isolati (13). La pressione selettiva costituita dalla esposizione ripetuta agli antibiotici ha causato la comparsa di meccanismi di resistenza batterici, che rendono difficoltoso il trattamento clinico delle infezioni urinarie. Poiché la presenza di chiari sintomi di infezione di regola motiva l'inizio di una terapia empirica precoce, la conoscenza della resistenza batterica dei comuni patogeni urinari sulla base delle indicazioni epidemiologiche locali, può for- nire le prime indicazioni utili al clinico per indirizzare la scelta del trattamento in attesa dei risultati del laboratorio relativi all'esame colturale e al profilo rilevato all'antibiogramma $(1,11)$.

\section{Presentazione dello Studio}

Nel presente studio, sono riportati i dati relativi alla sensibilità dei Gram-negativi isolati nel Laboratorio di Patologia Clinica e Microbiologia del Polo Oncologico/Dermatologico IFO nel periodo compreso tra ottobre 2000 e ottobre 2003. Le urinocolture sono state effettuate su 3650 campioni provenienti da 2835 pazienti ambulatoriali (77\% del totale delle afferenze) e da 815 pazienti ricoverati $(23 \%)$. Per l'isolamento batterico è stato utilizzato il metodo di semina su piastre a 
doppio settore (Agar sangue/Mac-Conkey-4 Metil-Umberriferilglucoronide, BIOLIFE) e incubazione a $37^{\circ} \mathrm{C}$ per $18-24$ ore.

In 593 campioni (16\%) è stata rilevata una crescita batterica uguale o superiore a $100.000 \mathrm{CFU} / \mathrm{ml}$ $\mathrm{e}$ si è proceduto alla identificazione biochimica $\mathrm{e}$ al test di sensibilità agli antibiotici mediante sistema VITEK 1 (bioMèrieux Italia). La tipizzazione ha rivelato la presenza di $E$. coli in 435 campioni urinari (73\%), di P. mirabilis in 57 (9.6\%), di $K$. pneumoniae in $53(8.9 \%)$, di $P$. aeruginosa in 27 (4.5\%). Altri Gram-negativi o Gram-positivi sono stati rinvenuti rispettivamente nel $3 \%$ e $1 \%$ dei campioni.

I valori di sensibilità ai differenti antibiotici sono riportati nella tabella 1 come percentuale di ceppi sensibili. L'analisi delle antibiotico-resistenze tra ceppi provenienti da pazienti ambulatoriali o da pazienti ricoverati presso i reparti di degenza non ha rivelato differenze significative, conseguentemente nella tabella sono riportati i dati relativi a tutti i campioni processati.

Tabella I. Sensibilità agli antibiotici espressi in valori percentuali delle specie microbiche isolate in 3650 campioni urinari nel periodo 2000-2003

\begin{tabular}{lcccc}
$\begin{array}{c}\text { Specie } \\
(\mathrm{N})\end{array}$ & $\begin{array}{c}\text { E. coli } \\
(435)\end{array}$ & $\begin{array}{c}\text { P. mirabilis } \\
(57)\end{array}$ & $\begin{array}{c}\text { K. pneumoniae } \\
(53)\end{array}$ & $\begin{array}{c}\text { P. aeruginosa } \\
(27)\end{array}$ \\
\hline Ac. Nalidixico & 82 & 78 & 85 & 0 \\
\hline Amikacina & 100 & 100 & 96 & 68 \\
\hline Amox/Ac-Clav & 84 & 88 & 92 & 0 \\
\hline Ampicillina & 62 & 65 & 0 & 0 \\
\hline Aztreonam & 96 & 96 & 98 & 46 \\
\hline Cefalotina & 88 & 75 & 87 & 0 \\
\hline Cefotaxime & 98 & 93 & 100 & 0 \\
\hline Ceftazidime & 97 & 96 & 100 & 61 \\
\hline Ciprofloxacina & 84 & 91 & 98 & 52 \\
\hline Fosfomicina & 97 & 60 & 83 & - \\
\hline Gentamicina & 93 & 89 & 98 & 52 \\
\hline Imipenem & 100 & 98 & 100 & 72 \\
\hline Nitrofurantoina & 98 & 0 & 74 & 0 \\
\hline Norfloxacina & 83 & 91 & 98 & 58 \\
\hline Piperacillina & 76 & 82 & 87 & 76 \\
\hline Ticarc/ac Clav & 87 & 100 & 92 & 64 \\
\hline Tobramicina & 96 & 96 & 98 & 54 \\
\hline Trimethoprin/sulf 78 & 66 & 94 & 0 \\
\hline
\end{tabular}

\section{CONCLUSIONI}

E. coli è la causa più frequente delle infezioni del tratto urinario rappresentando il $75-95 \%$ di tutti gli isolati seguito da altri germi appartenenti alla famiglia delle Enterobacteriaceae, come Klebsiella spp e Proteus spp (13). Negli ultimi 10 anni i patogeni delle vie urinarie hanno mostrato un aumento di resistenza a molti antibiotici con una diminuita sensibilità ai farmaci più comuni come ampicillina, amoxicillina/ac. clavulanico, trimethoprim/sulfametossazolo e fluorochinoloni
(5). Questo aumento di frequenza dei fenomeni di resistenza rende necessario lo sviluppo di dati epidemiologici locali per ottimizzare la scelta dei farmaci da adottare per la tempestiva introduzione di una terapia empirica.

In accordo con i dati della letteratura relativi a casistiche americane ed europee i nostri dati evidenziano anche nella nostra realtà locale l'insorgenza di resistenze ai farmaci maggiormente utilizzati per la terapia di infezioni urinarie $(2,5)$. Infatti, seppure in percentuali diverse, i germi Gram-negativi più frequentemente isolati, E. coli e P. mirabilis, hanno mostrato una significativa resistenza all'ampicillina: rispettivamente $38 \%$ e $35 \%$.

$K$. pneumoniae e $P$. aeruginosa sono risultati resistenti nel $100 \%$ dei casi allo stesso farmaco che, conseguentemente, non è indicato come trattamento empirico di scelta. Inoltre, abbiamo evidenziato, nel $22 \%$ di dei ceppi di E. coli e nel $33 \%$ dei ceppi di $P$. mirabilis una resistenza a trimethoprim/sulfametossazolo (SXT). Ciò è in sintonia con quanto descritto in altri Paesi dove peraltro il SXT è tuttora raccomandato come trattamento empirico di prima linea $(3,16)$.

Considerando che un farmaco di prima scelta non dovrebbe superare la soglia del $20 \%$ della frequenza di resistenza batterica, i fluorochinolonici potrebbero rientrare tra $\mathrm{i}$ farmaci adottabili per una terapia empirica (16). Nel nostro studio infatti la percentuale di resistenza ai fluorochinoloni è risultata del $16 \%$, similmente a quanto riscontrato in Spagna e Portogallo (5). Anche verso questi farmaci, comunque, è in atto un aumento progressivo delle resistenze batteriche, come emerge dai dati del SENTRY (8) che riporta un aumento delle resistenze di $E$. coli alla ciprofloxacina nei paesi Latino Americani. Per quanto riguarda la nitrofurantoina, la percentuale di resistenza di $E$. coli è del $2 \%$, analogamente a quella riportata recentemente da altri autori (6), nonostante questo chemioterapico sia utilizzato ormai da molti anni (16). La pressione selettiva indotta dall'esposizione agli antibiotici è particolarmente evidente in $P$. aeruginosa, di cui è comune l'identificazione di ceppi resistenti, soprattutto in ambito nosocomiale a causa dell'elevato numero di pazienti portatori di catetere vescicale a permanenza $(11,15)$. La produzione di slime, caratteristica di P. aerugino$s a$, può essere responsabile sia della facile trasmissione al paziente da parte degli operatori che dello sviluppo di antibiotico-resistenza anche verso i farmaci ritenuti maggiormente efficaci. Infatti, i germi nascosti nello slime, non sono raggiungibili da dosi efficaci di antibiotico (11).

$P$. aeruginosa, nella nostra casistica, risulta avere una elevata resistenza a ciprofloxacina (48\%) e ad imipenem (28\%). Questo dato riveste particolare 
interesse in quanto deriva da una casistica prevalentemente ambulatoriale (24 pazienti esterni vs 3 ricoverati) e dimostra che l'aumento generalizzato del consumo di antibiotici ha causato la diffusione di ceppi multi-resistenti di $P$. aeruginosa anche al di fuori dell'ambiente nosocomiale. Conseguentemente, anche in pazienti ambulatoriali ed in presenza di fattori predisponenti risulta particolarmente indicata l'esecuzione di una corretta urinocoltura e l'attesa dei risultati relativi alla identificazione del microrganismo responsabile e dell'antibiogramma, prima di instaurare una qualsiasi terapia empirica.

\section{BIBLIOGRAFIA}

1. Bassetti D, Bassetti M, Mantero E. Strategies for antibiotic selection in empirical therapy. Clin Microbiol Infect 2000; 6(Suppl.3): 98-100.

2. Dyer IE, Sankary TM, Dawson JA, Antibiotic resistance in bacterial urinary tract infections, 1991-1997. West J Med 1998; 169: 265-8.

3. Gupta K, Sahm DF, Mayfield D, Stamm WE. Antimicrobial resistance among uropathogens that cause community-acqired urinary tract in women; A nationwide analysis, Clin Infetct Dis 2001; 33: 89-94.

4. Howard AJ, Magee JT, Fitzgerald KA, Dunstan FDJ, Factors associated with antibiotic resistance in coliform organism from community urinary tract infection in Qales. J Antimicrob Chemother 2001; 47: 30513.

5. Kahlmeter G. The ECO-SENS project: A prospective, multinational multicentre epidemiological survey of the prevalence and antimicrobial susceptibility of urinary tract pathogens-interim report. J Antimicrob Chemother 2000; 46(Suppl. SI): 15-22.

6. Karlowsky JA, Tromsberry C, Jones ME, Sahm DF. Susceptibility of antimicrobial-resistant urinary Escherichia coli isolates to fluorochinolone and nitrofurantoin. Clin Infect Dis 2003; 36: 183-7.

7. Kelley A Gordon, Ronald N Jones. Susceptibility patterns of orally administered antimicrobials among urinary tract infection pathogens. Diag Microbiol Infect Dis 2003; 45: 295-301.

8. Mathai D, Jones RN, Pfaller MA, the SENTRY participant group North America. Epidemiology and frequency of resistance among pathoges causing urinary tract infections in 1,510 hospidalized patients: a report from the SENTRY antimicrobial surveillance program (North America). Diag Microbiol Infect Dis 2001; 40: 129-36.

9. Naber KG. Treatment options for acute uncomplicated cystitis in adults. J Antimicrob Chemother 2000; 46 (Suppl. SI): 23-7.

10. Navaneeth BV, Belwadi S, Suganthi N. Urinary pathogens' resistance to common antibiotics a retrospective analysis. Trop Doct 2002; 32: 20-2.

11. Nicolle LE. The chronic indwelling catheter and urinary infection in long term care facility residents. Infect Control Hosp Epidemiol 2001; 22: 316-21.

12. Nicolle LE. Resistant pathogens is urinary tract infection. J Am Geriatric Soc 2002; 50: S230-5.

13. Nicolle LE. Urinary tract infection: traditional pharmacologic therapies. Dis Mon 2003; 49: 111-28.

14. Ronald AR, Nicolle LE, Stamm E, et al. Urinary tract infection in adults: research priorities and strategies.
Int J Antimicrob Agents 2001; 17: 343-8.

15. Taneja N, Meharwal SK, Sharma M. Significance and characterization of pseudomonas from urinary tract specimens. J Commun Dis 2004; 36: 27-34.

16. Warren JW, Abrutyn E, Hebel JR, Johnson JR, Schaffer AJ, Stamm WE. Infectious Disease Society of America (IDSA). Guidelines for antimicrobial treatment of uncomplicated acute bacterial cystitis and acute pyelonephritis in women. Clin Infect Dis 1999; 29: 745-58.

\section{Maria Teresa Gallo}

Laboratorio di Patologia Clinica e

Microbiologia

Istituto San Gallicano, IRCCS;

Polo Dermatologico I.F.O.

Via Elio Chianesi 53 - 00144 Roma

Tel.: 0652666966 Fax: 0652666118

e-mail: gallo@ifo.it 